\title{
Achillessehnenrupturen bei Hunden und Katzen - allgemeine Therapiegrundsätze
}

\author{
Stefanie Klingler, Franck Forterre
}

Die Achillessehnenruptur ist beim Kleintier eine seltene Erkrankung mit einer guten Prognose. In der Therapie stellt die Immobilisation und die anschließende Mobilisation der Gliedmaße den wichtigsten Faktor dar, wobei in diesem Zusammenhang auch am meisten Komplikationen zu verzeichnen sind.

\section{Anatomie der Achillessehne}

Die Achillessehne besteht aus folgenden Muskeln [1, 2]:

- M. gastrocnemius

- M. flexor digitalis superficialis

- gemeinsame Endsehne des M. biceps femoris und des M. semitendinosus

Beim Hund beteiligt sich zusätzlich der M. gracillis [1] und bei der Katze zudem der M. soleus an der Bildung der Achillessehne [1].

\section{Ätiologie}

Als häufigster Grund einer akuten Achillessehnenruptur ist ein einmaliges direktes Trauma beschrieben [8].

Häufig handelt es sich um $[9,19]$ :

- Lazerationen durch scharfe Gegenstände

- Überlastungen des Muskel-SehnenApparats, z. B. durch Sprünge und Landen auf den Hinterbeinen

- Autounfälle

Chronische Verletzungen rühren bei Hunden eher von einer Überbeanspruchung, die zu einer chronischen Dehnung der Sehne und einer progressiven Verschlechterung führen [2]. Chronischdegenerative Veränderungen bei Katzen sind als Folge einer Tendinopathie oder Enthesiopathie beschrieben. Diese können zu einer akuten Schwäche der Achil- lessehne führen, wobei es zu einer akuten Ruptur durch ein triviales Trauma kommen kann [16].

\section{Einteilung der Rupturen}

Folgende Einteilung der Rupturen ist möglich [2]:

- partiell oder komplett

- akut oder chronisch

- offen oder geschlossen

\section{Symptomatik}

\section{Akute Verletzungen}

Bei der klinischen Untersuchung ist bei Rupturen, die durch ein akutes Trauma ausgelöst wurden, oft eine Schnittverletzung oder eine sonstige Hautwunde proximal des Tarsus vorhanden [2]. Zusätzlich belasten die betroffenen Tiere die Gliedmaße meist nicht [2] oder fußen bei kompletten Rupturen plantigrad auf. Bei einer partiellen Ruptur ist eine leichte bis moderate Hyperflexionsstellung zu sehen [9]. Bei einem intakten oberflächlichen Zehenbeuger kommt es zu einer gleichzeitigen Flexion der Zehen bei Belastung der Gliedmaße [2]. Bei einer Luxation der Fersenkappe gehen die Tiere intermittierend lahm, haben jedoch nur eine geringe Tarsushyperflexion [9].

\section{Chronische Verletzungen}

Bei chronischen Verlängerungen zeigen die Tiere einen mehr oder weniger plantigraden Gang [2].

\section{Diagnostik}

Zur Diagnose der Achillessehnenruptur sollte zuerst eine gründliche Anamnese erhoben werden [4], danach sollte eine allgemeine klinische und orthopädische Untersuchung erfolgen. Anschließend sind weiterführende Untersuchungen zur Diagnose von Avulsionsfrakturen,
Rupturen des M. gastrocnemius und Fremdkörpern möglich. Für eine genauere Diagnostik bieten sich Röntgenuntersuchungen in 2 Ebenen [9], Ultraschall [9] oder eine Kernspintomografie [18] an.

Als wichtigste Differenzialdiagnosen sind folgende Erkrankungen auszuschließen $[2,10]$ :

- Kalkaneusfraktur

- Ischiadicusverletzung

- kongenitale Hyperflexion

- Hypothyreose

- diabetische Neuropathie

\section{Untersuchung der Achillessehne}

Zur Kontrolle der Achillessehne wird das Knie in einer gestreckten Position gehalten, der Tarsus gebeugt und die Achillessehne palpiert [2]. Normalerweise stellt sich die Achillessehne als Strang dar. Bei einer Ruptur sollte man Unregelmäßigkeiten oder Schwellungen an der Achillessehne spüren. Bei einer kompletten Ruptur ist der Strang nicht mehr vorhanden, dafür sind die Sehnenenden oft palpierbar [9]. Zudem kann man den Tarsus bei gestrecktem Knie in eine Hyperflexionsstellung bringen [2]. Partielle Rupturen weisen oft nur eine Schwellung im Bereich der Läsion auf. Der Tarsus lässt sich nur in eine leichte bis moderate Hyperflexionsstellung bringen [9].

\section{Therapie}

Die Therapie der Achillessehne kann entweder konservativ oder chirurgisch erfolgen. Eine konservative Therapie kann durch Immobilisation des Sprunggelenks, Käfigruhe, Bewegungseinschränkung und/oder Physiotherapie erfolgen [15].

(weiter auf S. 22) 


\section{Studie der Kleintierklinik Bern}

\section{Studienpopulation}

An der Kleintierklinik des Tierspitals Bern wurden zwischen Januar 2005 und Juli 201523 Tiere mit einer Ruptur des Achillessehnenapparats vorgestellt. Dazu gehören 16 Hunde und 7 Katzen. Von den 16 Hunden waren 8 männlich und 8 weiblich. Insgesamt waren 7 Hündinnen und 3 Rüden kastriert. Das durchschnittliche Alter der Tiere lag bei 5,85 Jahren (1,8314,0 Jahre). Von der Achillessehnenruptur waren vorwiegend mittelgroße und große Hunde mit einem durchschnittlichen Gewicht von 23,38 kg (8-40 kg) betroffen. Zwei der Hunde wurden zur Jagd und einer als Arbeitshund unbekannter Nutzung eingesetzt. Ein Hund hatte anamnestisch bereits eine partielle Achillessehnenruptur an der kontralateralen Gliedmaße und ein anderer hatte eine Totalruptur an derselben Gliedmaße 4 Jahre zuvor.

Unter den 7 europäischen Kurzhaarkatzen waren 5 kastrierte Kater, eine kastrierte und eine intakte Katze. Das durchschnittliche Alter betrug 5,26 Jahre (3,510 Jahre) und das durchschnittliche Gewicht lag bei $5,38 \mathrm{~kg}(3,21-9,53 \mathrm{~kg})$. Zwei Katzen waren Wohnungskatzen und 5 Katzen waren Freigänger.

\section{Analyse}

Die Analyse erfolgte retrospektiv und deskriptiv in einer Gruppe für Hunde und einer für Katzen. Die Daten für die Studie wurden aus dem Polypoint, einem Datenerfassungsprogramm der Vetsuisse Fakultät Bern entnommen. Bei fehlenden Informationen über den Heilungsverlauf wurden die jeweiligen Privattierärzte und/oder die Besitzer telefonisch kontaktiert.

\section{Ätiologie, Klinik und Befunde}

Die Achillessehnenrupturen wurden in akute (< 48 Stunden), subakute (48 Stunden bis 3 Wochen) oder chronische Stadien ( $>3$ Wochen) eingeteilt ( $\vee$ Tab. 1). Ätiologie, Symptomatik, Lokalisation und Befunde der vorgestellten Tiere sind in $>$ Tab. 1 ersichtlich.

\section{Therapie}

Die Therapie erfolgte konservativ oder chirurgisch ( Tab.2). Die konservativ behandelten Hunde wurden entweder mit externer Ruhigstellung oder mittels interner Immobilisation durch einen Fixateur externe therapiert. Drei der 4 mit externer Ruhigstellung behandelten Hunde wurden im Median für 6 Wochen (2-8 Wochen) mit Verbänden mobilisiert. Ein Hund wurde nur mit Physiotherapie behandelt. Von den Hunden mit

Tab. 1 Achillessehnenrupturen im Tierspital Bern.

\begin{tabular}{|c|c|c|}
\hline Achillessehnenruptur & Anzahl der Hunde & Anzahl der Katzen \\
\hline \multicolumn{3}{|l|}{ Auftreten der Symptome } \\
\hline plötzlich & 14 & 5 \\
\hline schleichend & 2 & 2 \\
\hline \multicolumn{3}{|l|}{ Ätiologie } \\
\hline Ruptur ausgelöst durch Schnitt & 7 & 2 \\
\hline Ruptur ausgelöst durch Trauma anderer Art & 6 & 2 \\
\hline kein beobachtetes Trauma & 3 & 3 \\
\hline \multicolumn{3}{|l|}{ Symptome } \\
\hline Lahmheit & 16 & 7 \\
\hline Wunde & 5 & 4 \\
\hline plantigrades Auffußen & 4 & 7 \\
\hline tieferer Tarsus & 3 & 0 \\
\hline Schwellung & 5 & 0 \\
\hline Muskelatrophie & 1 & 0 \\
\hline \multicolumn{3}{|l|}{ Lokalisation } \\
\hline links & 8 & 3 \\
\hline rechts & $10^{*}$ & 4 \\
\hline \multicolumn{3}{|l|}{ Diagnose } \\
\hline akute, partielle, offene Ruptur & 2 & 0 \\
\hline akute, partielle, geschlossene Ruptur & 0 & 1 \\
\hline akute, komplette, offene Ruptur & 4 & 3 \\
\hline subakute, partielle, geschlossene Ruptur & 4 & 2 \\
\hline chronische, partielle, geschlossene Ruptur & 6 & 0 \\
\hline chronische, komplette, offene Ruptur & 0 & 1 \\
\hline
\end{tabular}

alleiniger interner Fixation konnten 2 Tiere nachverfolgt werden. Diese wurden für 3 und 6 Wochen immobilisiert, dann mit Verbänden für 4 und 8 Wochen progressiv mobilisiert.

Von den 9 chirurgisch therapierten Hunden wurden 7 mit einer Sehnennaht genäht. Fünf erhielten zur Immobilisation einen Fixateur externe, ein Hund erhielt eine calcaneotibiale Stellschraube und ein Hund eine Kombination beider 
Tab. 2 Therapie, Komplikationen und Heilungsverlauf der Achillessehnenrupturen der am Tierspital Bern vorgestellten Tiere.

\begin{tabular}{lll}
\hline Achillessehnenruptur & Anzahl der Hunde & Anzahl der Katzen \\
\hline Therapie & & 0 \\
\hline konservativ mittels externer Ruhigstellung & 4 & 0 \\
\hline $\begin{array}{l}\text { konservativ mittels interner Fixierung mit } \\
\text { Fixateur externe }\end{array}$ & 3 & 6 \\
\hline $\begin{array}{l}\text { chirurgisch mit Sehnennaht und interner } \\
\text { Fixation }\end{array}$ & 7 & \\
andere chirurgische Technik & 2 & 1 \\
\hline Komplikationen & & 2 \\
\hline minimal & 5 & 2 \\
\hline schwerwiegend & 4 & 2 \\
\hline keine & 3 & 0 \\
\hline minimal und schwerwiegend & 2 & 1 \\
\hline keine Informationen vorhanden & 2 & 2 \\
\hline klinisches Endergebnis & 1 & 3 \\
\hline gut & 15 & 2 \\
\hline schlecht & 0 & 2 \\
\hline keine Informationen vorhanden & & 2 \\
\hline
\end{tabular}

Maßnahmen. Diese Tiere wurden im Median für 5,5 Wochen (4-9 Wochen) immobilisiert. Ein Hund war nicht nachverfolgbar. Bei den 2 Hunden, die ohne Sehnennaht therapiert wurden, lag eine Luxation des M. flexor digitalis superficialis und eine Avulsion der Fersensehnenkappe vor. Beide wurden mit Pins fixiert. Die Gliedmaße wurde in diesen beiden Fällen nicht immobilisiert. Alle chirurgischen Patienten wurden progressiv mit einer Kombination aus Schiene und/oder Cast und/oder Verbänden im Median für 7 Wochen (0-33 Wochen) progressiv mobilisiert.

Alle Katzen wurden chirurgisch versorgt ( $\triangleright$ Tab.2). Fünf Katzen wurden nur mittels Sehnennaht therapiert. Ein knöcherner Ausriss wurde, wie bereits beschrieben, mittels Knochentunneln und einer Sehnennaht therapiert. Bei der Katze mit der chronischen vollständigen Ruptur wurde eine Tarsusarthrodese vorgenommen. Die 3 partiellen Rupturen wurden mittels calcaneotibialer Stellschraube ( $\triangleright$ Abb.4), die anderen 4 mittels Fixateur externe im Median für 5 Wochen (4-16 Wochen) komplett immobilisiert. Darauf folgte bei allen eine progressive Mobilisation mittels Schiene und Verband für im Median 3,5 Wochen (0-10 Wochen).

\section{Komplikationen}

Die am Tierspital Bern aufgetretenen Komplikationen wurden in minimal und schwerwiegend unterteilt ( $\triangleright$ Tab.2).

Minimale Komplikationen:

- Verbandskomplikationen

- Schienenbruch

- Reruptur der Sehne

- oberflächliche Pintraktinfektionen

Schwerwiegende Komplikationen:

- Osteomyelitis

- Schraubenbruch

- Pininstabilität

- Allgemeininfektionen

- Trauma mit Entfernung des Fixateur externe

- Infektionen mit multiresistentem Staphylococcus aureus

- schlechtes klinisches Endergebnis

\section{Diskussion}

Obesitas wird als prädisponierender Faktor beschrieben [7]. Diesbezüglich ist am Tierspital Bern eine Katze aufgefallen. Die Katze war 4 Jahre und 2 Monate alt, wog 9,53 kg und erlitt einen partiellen Abriss der Achillessehne ohne beobachtetes Trauma. Im Ultraschall waren mineralisierte Bereiche $\mathrm{zu}$ sehen, weshalb auf ein chronisches Geschehen geschlossen werden konnte. Es ist beschrieben, dass vor allem alte Katzen mit Übergewicht und chronisch-degenerativen Veränderungen eine atraumatische Achillessehnenruptur entwickeln können [16]. Vermutlich könnte es aber durch das massive Übergewicht der Katze bereits frühzeitig in diesem Alter zu einer solchen Schwächung der Sehne und Degeneration mit Ruptur gekommen sein.

In der Literatur wird von konservativen Therapien meist nur im Zusammenhang mit missglückten Therapieversuchen gesprochen $[6,13]$. Die am Tierspital Bern mit konservativer Therapie behandelten Hunde waren soweit nachvollziehbar alle gut abgeheilt. Die missglückten konservativen Therapien, die in den genannten Studien angesprochen wurden, könnten auf eine zu wenig konsequente Immobilisation zurückzuführen sein $[6,13]$. Unter Umständen könnte auch eine falsche Indikation für eine konservative Therapie gewählt worden sein. Muskel-

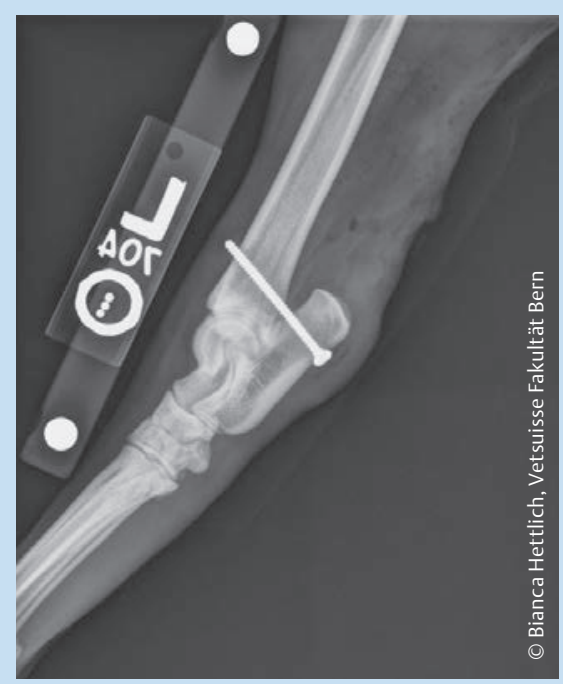

Abb. 4 Postoperatives Röngten: Immobilisierung des Gelenks mit einer calcaneotibialen Stellschraube. 
zerrungen, partielle Muskelrupturen, partielle Rupturen einzelner Sehnenanteile und chronische partielle Rupturen scheinen gute Kandidaten für eine konservative Therapie zu sein.

Die Tendenz, dass die meisten Komplikationen im Zusammenhang mit der Immobilisierung auftreten $[2,11]$, konnte auch in dieser Studienpopulation gesehen werden. Der größte Anteil umfasste Druckstellen bei 4 Tieren gefolgt von oberflächlichen Pintraktinfektionen bei 2 Tieren. In der Klinik sollte deshalb ein besonderes Augenmerk auf der guten Polsterung, den guten Sitz des Verbands und gegebenenfalls der Schiene liegen. Beim Umgang mit dem Fixateur externe sollte auf eine gute Hygiene geachtet und gegebenenfalls vermehrt mit desinfizierenden Salben, zum Beispiel einer Bedatine ${ }^{\circledR}$ Salbe, gearbeitet werden.
Die adipöse Katze erlitt einen Bruch der calcaneotibialen Stellschraube ohne Reruptur der Sehne. Es wird vermutet, dass die Schraube aufgrund der erhöhten Belastung gebrochen ist. Unter Umständen sollte bei obesen Tieren daher eine zusätzliche Immobilisation verwendet werden. Falls möglich, könnte zusätzlich ein Fixateur externe eingesetzt werden oder eine zusätzliche Schiene angewendet werden.

Haustiere mit einer Achillessehnenruptur haben eine gute Prognose $[2,9,11]$. Diese Resultate konnten auch bei der Hundepopulation am Tierspital Bern gesehen werden, da alle nachvollziehbaren Hunde ein gutes klinisches Endergebnis zeigten. Von 5 nachverfolgbaren Katzen zeigten 2 ein schlechtes klinisches Endergebnis.
In 2 Publikationen ist beschrieben, dass es keine Auswirkungen auf das klinische Endergebnis hat, ob es sich ursprünglich um eine offene oder geschlossene, eine akute, subakute oder chronische, eine partielle oder komplette Ruptur handelt $[2,11]$. Meist sei ein gutes klinisches Endergebnis zu erwarten $[2,9,11,19]$. Zusätzlich scheint auch die Technik der chirurgischen Therapie keine Auswirkungen auf das klinische Endergebnis zu haben, solange eine gute Immobilisation und somit eine Entlastung der Sehne gewährleistet werden kann [9]. Diese Tendenzen konnten auch bei den Patienten des Tierspitals Bern gesehen werden. Wichtig scheint in jedem Fall eine adäquate Beurteilung aller relevanten Faktoren und eine angemessene Diagnostik der Läsion, um eine optimale Therapie mit einer erfolgreichen Immobilisation erzielen zu können.

\section{Immobilisation des Sprunggelenks}

Für eine chronische Ruptur wird eine konservative Therapie empfohlen [9]. Bei einer partiellen Ruptur kann eine konservative Therapie mit Immobilisation versucht werden, allerdings sind die Ergebnisse oft unbefriedigend [5]. Im Rahmen der konservativen Therapie wird eine Immobilisation des Sprunggelenks empfohlen. Dazu existieren mehrere Möglichkeiten, die alle den Zweck verfolgen, die Achillessehne vor einer weiteren Schädigung durch Zug zu bewahren. Die Gliedmaße kann mittels Fixateur externe, calcaneotibialer Stellschraube, Gipsverband, Schiene oder anderen Verbänden immobilisiert werden. Dabei wird im Verlauf der Behandlung meist eine Kombination aus den verschiedenen Methoden angewendet. Die calcaneotibiale Stellschraube und der Fixateur externe müssen in einem operativen Eingriff platziert werden. Für eine optimale Heilung sollte der Tarsus mindestens 3 Wochen immobilisiert, danach über weitere 3-4 Wochen progressiv mobilisiert werden. Je nach klinischem Bild muss die Gliedmaße auch länger immobilisiert werden [17]. 


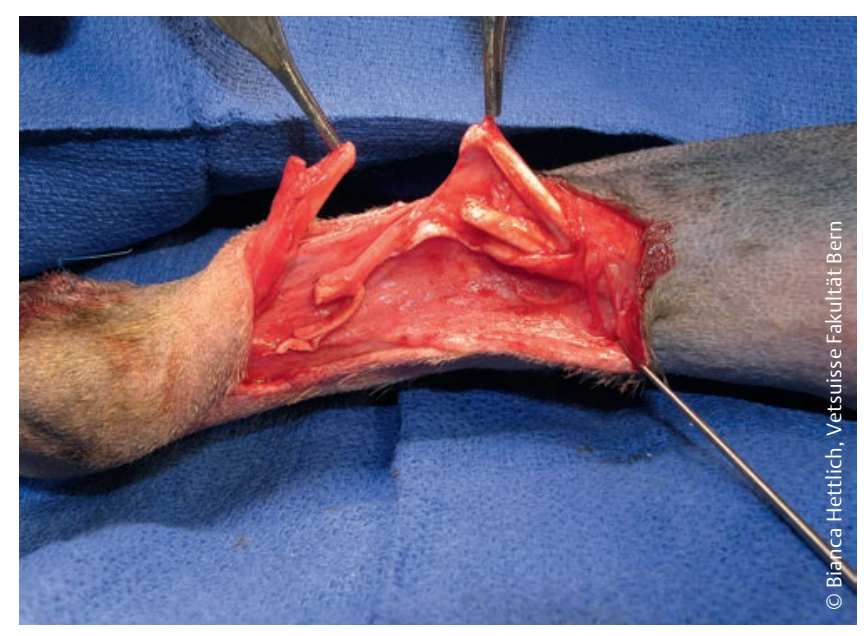

Abb. 1 Intraoperative Visualisierung der 3 Sehnenanteile.

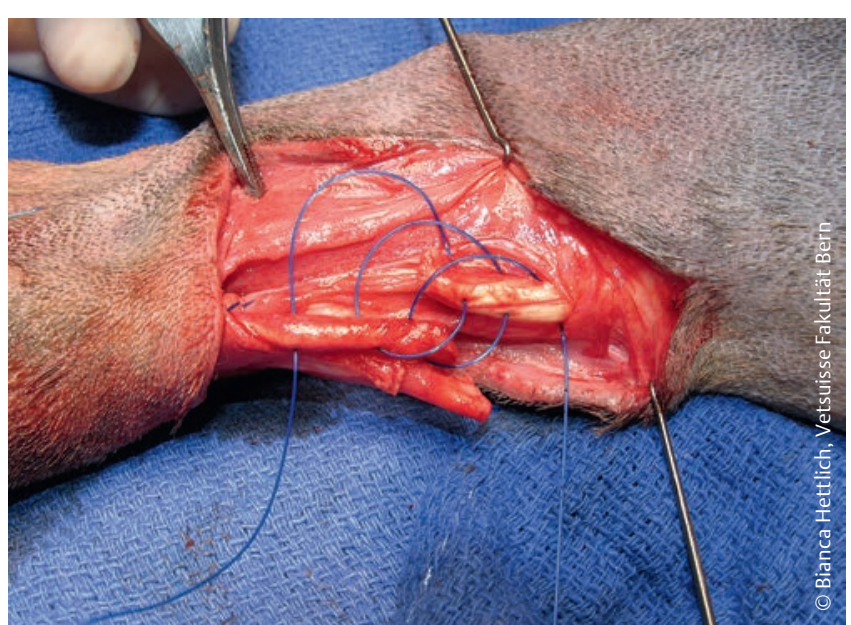

Abb. 2 Naht eines Sehnenstumpfs mit einer „three-loop-pulley“-Naht.

\section{Chirurgischer Eingriff an der Sehne}

Als Indikation für eine chirurgische Therapie werden akute, komplette Rupturen, offene Sehnenverletzungen und eine Luxation der Fersenkappe beschrieben [9]. Ein Zugang von kaudolateral ist empfohlen [2].
Bei einer offenen Ruptur sollte immer ein separater chirurgischer Zugang angestrebt werden, da sonst eine Ausbreitung des Narbengewebes zur Hautwunde möglich ist [17].
Um die Sehnenenden zu schützen, sollte mit Haltenähten, Nadeln oder Kirschnerdrähten entfernt von diesen gearbeitet werden, damit das Gewebe nicht zusätzlich traumatisiert wird. Diese sind auch hilfreich, um Zug auf die Sehne auszuüben [17]. 


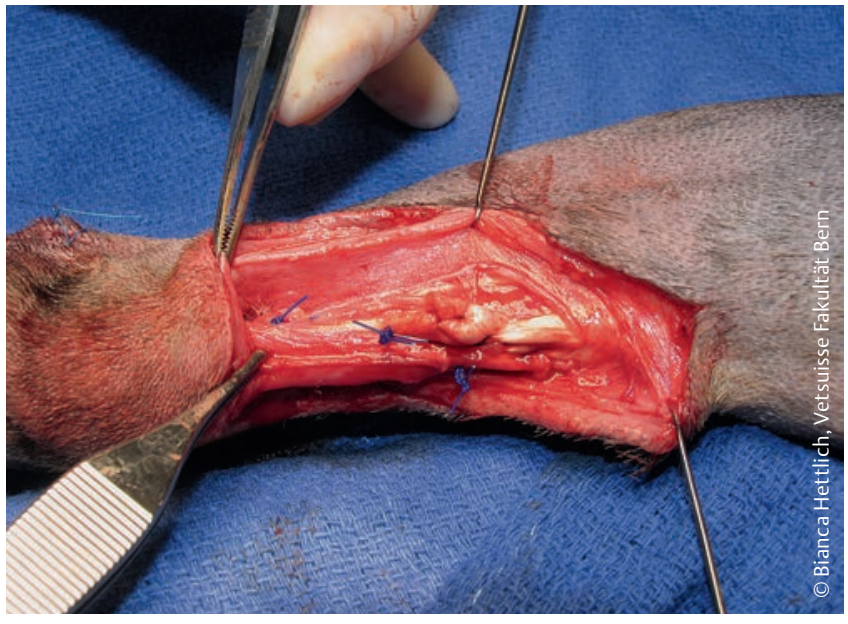

Abb. 3

Einzeln vernähte Anteile der Achillessehne.

Bei akuten Rupturen sind alle 3 Sehnenteile einzeln $\mathrm{zu}$ identifizieren $(\boldsymbol{A} \mathbf{A b} \mathbf{b} . \mathbf{1})$ [2], zu debridieren [17] und die gerissenen Anteile separat zu vernähen ( $\downarrow$ Abb.3), um eine möglichst gute Rekonstruktion der Sehne zu erreichen [2]. Eine Streckung und Beugung der umliegenden Gelenke kann bei der Adaption der Sehnenstümpfe hilfreich sein [17].

Es können folgende Nahttechniken verwendet werden $[2,17]$ :

- „three-loop-pulley“-Naht ( $\bullet$ Abb. 2)

- Fern-nah-nah-fern-Naht

- Bunell-Mayer-Naht

- Sehnennaht nach Kleinert

- „locking-loop“-Naht (Kessler-Naht)

- Krackow-Naht

Ein nicht [2] oder langsam resorbierbares monofiles Nahtmaterial wird empfohlen [17]. Je nach Größe der Sehne wird Nahtmaterial der Größe 4-0 bis 0 empfohlen [2]. Nach den Sehnennähten sollte nach Möglichkeit das Paratendineum vernäht werden, damit die Gleitfähigkeit der Sehne möglichst erhalten bleibt [1].

Bei chronischen Rupturen können die einzelnen Sehnenstümpfe nicht mehr identifiziert werden. Die fibröse Sehne wird freigelegt und es wird vom Zentrum der Fibrose her gerade so viel Narbenmaterial exzidiert, dass der Beugesehnenapparat in physiologischer Haltung des Knies und leicht gestrecktem Tarsus eine mäßige Spannung aufweist [2]. Falls die Sehnenenden aufgrund der Retraktion nicht mehr adaptierend vernäht werden können, sollte eine Sehnen- transplantation mit einem Stück Fascia lata angestrebt werden [1].

Bei einem knöchernen Ausriss der Achillessehne wird die Sehne mit einer der oben beschriebenen Nahttechniken genäht. Zudem werden Löcher in den proximalen Kalkaneus gebohrt und die Sehne, indem der Faden durch die Bohrlöcher geführt und verknotet wird, fixiert [2]. Immobilisation der Gliedmaße unabdingbar, um eine Reruptur und Lückenbildung zu verhindern [9].

Zur Immobilisation können die verschiedenen Techniken verwendet werden, die auch für die konservative Immobilisation verwendet werden.

\section{Komplikationen}

Bei der Therapie der Achillessehnenruptur können verschiedene Komplikationen auftreten. Diese stehen häufiger mit der Immobilisation im Zusammenhang als mit der Reparatur des Defekts selbst $[2,11]$.

Im Zusammenhang mit der Reparatur des Defekts selbst können folgende Komplikationen auftreten [1, 2]:

- Reruptur der Achillessehne

- Narbenbildung mit Tenodese und daraus folgendem Verlust der Gleitfähigkeit

- Einschränkung der Gleitfähigkeit durch eine Nekrose des Paratendineums
Für den Heilungsverlauf ist eine rigide
- Bildung von exzessivem Narbengewebe, Nahtdehiszenz und/oder Nekrose

- Sehnenverlängerung aufgrund zu früher Belastung, Trauma, unphysiologischer Schienung oder fehlerhafter Heilung

- Sehnenverkürzung infolge missglückter oder nicht erfolgter Sehnenbehandlung

- Fistelbildung

Die Komplikationen, die in Verbindung mit der Immobilisation des Gelenks auftreten, lassen sich in 4 Gruppen einteilen:

- Muskelatrophie aufgrund der Immobilisation des Tarsalgelenks

- Zusammenhang mit einem Fixateur externe $[1,2,11]$

- Lockerung

- Bruch des Pins

- Metatarsalfrakturen durch die Pins

- Bruch der Verbindungsstange

- Pintraktinfektionen und Osteomyelitis, die in Zusammenhang mit dem Pin gebracht werden konnten

- Zusammenhang mit calcaneotibialer Stellschraube [2]

- Lockerung

- Ausriss

- Schraubenbruch

- Verbandskomplikationen, inkl. Schiene und Cast $[1,2,11]$

- Hautirritationen

- Druckstellen

- Schwellungen

- Wunden

- Schienenbruch 


\section{Prognose und Diskussion}

Die Prognose einer Achillessehnenruptur ist für Hunde und Katzen, die als Haustiere gehalten werden, gut $[2,9,11]$. Arbeitshunde konnten in einer Studie in $70 \%$ der Fälle wieder eingesetzt werden [19].

\section{Online zu finden unter}

http://dx.doi.org/10.1055/s-0042-111780

\section{Literatur}

1 Bonath KH, Prieur WD. Kleintierkrankheiten: Band 3: Orthopädische Chirurgie und Traumatologie. Stuttgart: Eugen Ulmer; 1998: 64-77, 157-153, 697-713

2 Cervi M, Brebner N, Liptak J. Short- and long-term outcomes of primary Achilles tendon repair in cats: 21 cases. Vet Comp Orthop Traumatol 2010; 23 (5): 348-353
6 Guerin S, Burbidge H, Firth E et al. Achilles tenorrhaphy in five dogs: a modified surgical technique and evaluation of a cranial half cast. Vet Comp Orthop Traumatol 1998; 11: 205-210

7 Harasen G. Ruptures of the common calcaneal tendon. Can Vet J 2006; 47 (12): 1219-1220

8 King M, Jerram R. Achilles tendon rupture in dogs. Compend Cont Ed Pract Vet 2003; 25: 613-621

9 Kramer M, Schimke E, Gerwing M et al. Achillessehnenerkrankungen bei Hund und Katze. Tieraerztl Prax 1998; 26: 238-246

10 Nelson RW, Couto CG. Innere Medizin der Kleintiere. 2. Aufl. München: Urban und Fischer/Elsevier; 2010: 1140-1141

11 Nielsen C, Pluhar GE. Outcome following surgical repair of achilles tendon rupture and comparison between postoperative tibiotarsal immobilization methods in dogs: 28 cases (1997-2004). Vet Comp Orthop Traumatol 2006; 19: 246-249
15 Scheidl I, Sock K. Retrospektive Studie über Achillessehnenrupturen bei Hund und Katze: Fallbesprechungen und statistische Auswertung (2010). Im Internet: www. vetmeduni.ac.at/hochschulschriften/ diplomarbeiten/AC08340066.pdf; Stand 8. Mai 2016

16 Scott HW, McLaughlin R. Orthopädie bei der Katze: Erkrankungen und Therapie des Bewegungsapparats. Hannover: Schlütersche; 2008: 106, 254-260

17 Tobias KM, Jonston SA. Veterinary Surgery: Small Animal. Volume 1. St. Louis: Elsevier/ Saunders; 2012: 1124-1134

18 Weinstabl R, Stiskal P, Neuhold A et al. [MR and ultrasound study of Achilles tendon injury]. Unfallchirurgie 1992; 18 (4): 213-217

19 Worth AJ, Danielsson F, Bray JP et al. Ability to work and owner satisfaction following surgical repair of common calcanean tendon injuries in working dogs in New Zealand. N Z Vet J 2004; 52 (3): 109-116
3 Corr SA, Draffan D, Kulendra E et al. Retrospective study of Achilles mechanism disruption in 45 dogs. Vet Rec 2010; 167 (11): 407-411

4 Fahie MA. Healing, diagnosis, repair, and rehabilitation of tendon conditions. Vet Clin North Am Small Anim Pract 2005; 35 (5): 1195-1211

5 Fossum TW. Chirurgie der Kleintiere. 2. Aufl. München: Urban und Fischer/Elsevier; 2009: 1132, 1377-1385
12 Piermattei DL, Flo GL, DeCamp CE. Brinker, Piermattei and Flo's Handbook of Small Animal Orthopedics and Fracture Repair. $5^{\text {th }}$ ed. St. Louis, Missouri: Saunders/Elsevier: 2006; 674-678

13 Reinke JD, Mughannam AJ, Owens JM. Avulsion of the gastrocnemius tendon in 11 dogs. J Am Anim Hosp Assoc 1993; 29 (5): 410-418

14 Salomon FV, Geyer H, Gille U. Anatomie für die Tiermedizin. 3. Aufl. Stuttgart: Enke; 2008: 157-158, 223-234

\section{Stefanie Klingler}

Mannenriedstr. 5

3074 Muri, Schweiz

klinglerstefanie@yhaoo.de

\section{Prof. Dr. Franck Forterre}

Dipl. ECVS

Department für klinische Veterinärmedizin Vetsuisse Fakultät Bern

Länggassstr. 128

3012 Bern, Schweiz 